\title{
EMPRESAS NO LUCRATIVAS Y DESARROLLO
}

\section{Clara Caselli}

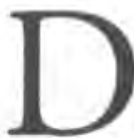

esde hace muchos años, el fenómeno de las iniciativas sin fines de lucro ha sido investigado por la John Hopkins University:' inicialmente, la investigación abarcaba los paises más desarrollados, pero ahora incluye también países en transición y en desarrollo. En el último reporte que ha sido publicado en el 2003, ${ }^{2}$ los países en vías de desarrollo son catorce y forman un conjunto significativo.

El último reporte confirma la extraordinaria relevancia del sector en el mundo (tabla 1), pero también las profundas diferencias entre país y país, por ejemplo, desde el punto de vista de la incidencia sobre el total de la población activa se va del $14,4 \%$ de los Países

1 Véase del autor SALAmon, Anheier: Il settore non profit in un confronto internazionale: profili e tipi (Università Cattolica del Sacro Cuore, Centro di ricerche sulla cooperazione, Quaderni occasionali, n.0 3, 1994); Il settore non profit in una prospettiva comparata. Una panoramica (Universita Cattolica del Sacro Cuore, Istituto per la ricerca sociale, Quaderni occasionali, n. ${ }^{\circ}$ 6, 1994); Global Civil Sociery, Dimensions of the Non Profit Sector (Baltimore: The Johns Hopkins Center for Civil Society Studies, MD, 1999), y Sılumon, L. M., S. W. Sokolowsкr, R. List, Global Civil Society. An Overview (Baltimore: The Johns Hopkins Center for Civil Society Studies, MD, 2003).

2 Véase Snlamon, Sokolowki, List. Global Civil Society. An Overview, 2003. 
Bajos al $0,4 \%$ de México y entre los países en desarrollo se va del máximo de Argentina, donde la incidencia es del 4,8\% (más que España, Japón e Italia), al mínimo de México.

Igualmente, muy variada es la incidencia del voluntariado: del $75 \%$ de Suecia y Tanzania al 2,8\% de Egipto. En cuanto concierne a las actividades desarrolladas, en países muy diferentes entre ellos por grado de desarrollo y cultura la prestación de servicios directos a la población prevalece sobre las actividades de expresión: el Perú tiene el porcentaje más alto, seguido por Bélgica, Israel, Irlanda, Pakistán, Corea y Estados Unidos, mientras que países como Suecia, Eslovaquia, Noruega se caracterizan por una baja incidencia. En las formas de financiamiento prevalece por doquier la venta directa de servicios, pero tal connotación caracteriza en mayor medida a países subdesarrollados: en los primeros puestos encontramos a Filipinas, México, Kenia, Brasil, Argentina, Corea, Colombia, Perú.

Comoveremos másadelante, el grado dedesarrolloes, sinduda, uno de los factores explicativos de las diferencias que se encuentran, pero no es el único. Desde este punto de vista, la investigación de la John Hopkins University ha realizado una tentativa interesante para individualizar los cluster.

Los países han sido primero subdivididos por grado de desarrollo. Entre los desarrollados se han identificado cuatro cluster:

- Modelo anglosajón: Se trata de países donde la presencia del sector no lucrativo es más relevante, ligada a una sólida tradición histórica; la presencia de voluntarios es significativa, pero no mucho respecto del promedio mundial; la orientación es hacia actividades de servicio (salud, educación, etc.) y los recursos derivan sobre todo de la venta de servicios, aunque no son irrelevantes las aportaciones públicas. 
Tabla 1: Dimensiones del sector no lucrativo en 35 paises del mundo (1995-98)

\begin{tabular}{|c|c|}
\hline Facturado & 1300000 millones de dólares \\
\hline Facturado / PBI & $5,1 \%$ \\
\hline Ocupados & 39,5 millones \\
\hline \multicolumn{2}{|l|}{ de los cuales: } \\
\hline Dependientes & 21,8 millones \\
\hline Voluntarios" & 12,6 millones \\
\hline $\begin{array}{l}\text { \% de los ocupados sobre la } \\
\text { población económicamente activa }\end{array}$ & $4.4 \%$ \\
\hline $\begin{array}{l}\% \text { de los ocupados sobre } \\
\text { la ocupación del sector público }\end{array}$ & $46 \%$ \\
\hline $\mathrm{N} .{ }^{\circ}$ voluntarios" & 190 millones \\
\hline $\begin{array}{l}N \text { N. voluntarios / población } \\
\text { adulta } x 1000\end{array}$ & 221 \\
\hline
\end{tabular}

- El número de voluntarios es calculado en términos equivalentes, ello es dividiendo al nủmero de horas de trabajo en conjunto trabajadas por los voluntarios por aquellas de un trabajador dependiente a tiempo completo.

"El número de voluntarios es calculado en términos absolutos, prescindienđo del nùrnero de horas dedicadas a las actividades de voluntariado.

Fuente: Johns Hopkins Comparative Nonprofit Sector Project

- Modelo nórdico: el peso del sector no lucrativo es alto, pero inferior al resto de países europeos y, en gran parte, basado en voluntariado y no orientado a los servicios. Este modelo se explica con las características del modelo del estado de bienestar adoptado tradicionalmente por estos países, que ha tratado de responder de modo directo a las necesidades sociales, dejando entonces espacio a la formación de iniciativas no lucrativas con características de expresión y de advocacy. 
- Modelo europeo de participación en el sistema de bienestar: en los otros países europeos, donde la intervención pública a menudo ha recorrido a la construcción de formas de partenariado con la sociedad civil (según modelos de subsidiariedad), se ha afirmado un no profic sólido y arraigado - también por la solidez de sus orígenes históricos-, con una fuerte presencia de trabajo dependiente, orientación a los servicios (educación, asistencia y salud) y significativa importancia de la intervención pública.

- Modelo asiárico de los países emergentes: el peso del sector no lucrativo es decididamente menor, por su más reciente formación y en general la falta de una actitud favorable por parte de las autoridades públicas, que son fuertemente burocratizadas, y está orientado a los servicios, de cuya venta provienen la mayor parte de los recursos utilizados.

En el grupo de los países en vías de desarrollo han sido identificados tres cluster principales (el cuarto es en realidad un agregado muy heterogéneo para poder ser considerado como tal y se prefiere no incluirlo en la descripción). Aquí las diferencias son esencialmente de tipo geográfico y reflejan los diferentes grados de desarrollo (ver tabla 2 ).

- Modelo latinoamericano: la presencia del sector no lucrativo es significativa, con una modesta presencia de voluntarios (bajo este aspecto existen, pero diferencias entre los países) y una predominante orientación a los servicios. Del punto de vista de los recursos empleados, la venta de los servicios alcanza el valor más alto en absoluto y revela una cierta 
capacidad de autofinanciamiento, que deriva también de la incapacidad del estado de satisfacer necesidades primarias; un cierto peso tienen las donaciones que provienen de los socios $y$, muy a menudo, del exterior.

- Modelo africano: también en este continente el sector no lucrativo tiene una cierca difusión, como una herencia de una tradición histórica no irrelevante, y tiene fuertes connotaciones de voluntariado. Las condiciones económicas hacen más difícil la venta de servicios al mercado y por lo tanto las contribuciones públicas y las donaciones ocupan un rol importante.

- Modelo de los países en transición: un pasado en el cual el rol del estado ha sido dominante, sino exclusivo y hostil a las expresiones de la sociedad civil, sobre todo si ellas tenían connotaciones religiosas, es el origen de la muy escasa difusión del sector no lucrativo. Lo que nace se dirige por lo tanto al desarrollo de actividades expresivas más que a la producción de servicios dirigidos al mercado: el sector no lucrativo tolerado era, en efecto, aquel con finalidades recreativas y profesionales, sensible a las influencias del régimen. La paradójica relevancia de las donaciones depende probablemente del proceso de desmembración de los grandes conglomerados públicos que ha provocado la organización al exterior de los servicios de tipo recreativo y asistencial que eran antes erogados directamente y en forma gratuita. 


\section{CLARA CASELLI}

Tabla 2: Comparación entre grupos de paises

\begin{tabular}{|c|c|c|c|c|c|c|}
\hline \multirow[t]{2}{*}{ Cluster } & \multirow{2}{*}{$\begin{array}{l}\text { \% Población } \\
\text { económica- } \\
\text { mente activa }\end{array}$} & \multirow[t]{2}{*}{ Voluntarios } & \multirow[t]{2}{*}{$\begin{array}{c}\text { Orientación } \\
\text { actividad }\end{array}$} & \multicolumn{3}{|c|}{ Financiamiento } \\
\hline & & & & $\begin{array}{c}\text { Ventas } \\
\text { Senvicios }\end{array}$ & Público & Donaciones \\
\hline Mundo & $4,4 \%$ & $36,4 \%$ & servicios & $53,4 \%$ & $34,9 \%$ & $11,7 \%$ \\
\hline Anglosajón & $8,2 \%$ & $36,6 \%$ & servicios & $54,6 \%$ & $36,1 \%$ & $9,3 \%$ \\
\hline Nórdico & $6,5 \%$ & $63,1 \%$ & expresión & $59,4 \%$ & $39,3 \%$ & $7.3 \%$ \\
\hline $\begin{array}{l}\text { Europeo } \\
\text { participativo }\end{array}$ & $7,8 \%$ & $29,5 \%$ & servicios & $35,4 \%$ & $57,6 \%$ & $7,0 \%$ \\
\hline $\begin{array}{l}\text { Asiático } \\
\text { emergente }\end{array}$ & $3,3 \%$ & $24,2 \%$ & servicios: & $61,8 \%$ & $34,8 \%$ & $3,5 \%$ \\
\hline $\begin{array}{l}\text { Paises en } \\
\text { desarrollo y } \\
\text { en transición }\end{array}$ & $1,9 \%$ & $36,8 \%$ & servicios & $62,3 \%$ & $21,6 \%$ & $16,1 \%$ \\
\hline América Latina & $2,3 \%$ & $30,4 \%$ & servicios & $74,4 \%$ & $15,3 \%$ & $10,3 \%$ \\
\hline Africa & 2,5 & $52,0 \%$ & servicios & $55,3 \%$ & $25,3 \%$ & $19,4 \%$ \\
\hline $\begin{array}{l}\text { Paises en } \\
\text { transición }\end{array}$ & $1,1 \%$ & $36,4 \%$ & expresión & $49,0 \%$ & $31,5 \%$ & $19,5 \%$ \\
\hline
\end{tabular}

Fuente: Johns Hopkins Comparative Nonprofit Sector Project

\section{El non profit en el Perú}

Una investigación realizada por la Universidad del Pacífico hace unos años ${ }^{1}$ nos permite colocar al Perú en el contexto internacional (rabla 3).

1 Portochrrero, F; C. SAnborn. "Entre el estado y el mercado: definiendo el sector sin fines de lucro en el Perún. Apuntes 43, n.², 1999. 
EMPRESAS NO LUCRATTVAS Y DESARROLLO

Tabla 3: El sector no lucrativo en el Perú (1995)

\begin{tabular}{|l|c|}
\hline Facturado & 1200 millones de dólares \\
\hline Facturado / PBI & $2 \%$ \\
\hline Ocupados & 150000 \\
\hline \multicolumn{1}{|c|}{ de los cuales: } & 126988 \\
Dependientes & 26400 \\
\hline Voluntarios & $2,9 \%$ \\
\hline \% de los ocupados sobre & \\
la póblación económicamente activa & $20 \%$ \\
\hline \% de los ocupados sobre & \\
la ocupación del sector público & \\
\hline
\end{tabular}

Fuente; Johns Hopkins Comparative Nonprofit Sector Project

Los datos, a pesar del hecho que se refieren al 1995, son muy interesantes: hay que subrayar que, por la insuficiente confiabilidad de los datos debido al alto nivel de informalidad, se consideraron 49430 organizaciones, entre las más de 110000 que se encontraron, por lo tanto, el fenómeno es, sin duda, subestimado en comparación con países que tienen sistemas estadísticos más confiables.

De todas maneras, la importancia del sector no lucrativo en términos de PBI es inferior al promedio mundial, pero alta en comparación con los demás países de América Latina (con la excepción de Argentina). Lo mismo se puede decir por lo que se refiere a la ocupación y al empleo de voluntarios: el sector no lucrativo emplea a más trabajadores que muchos sectores privados de punta como el minero y el pesquero. Desde este punto de vista, es interesante también mencionar un dato adicional que no está en la tabla, es decir, el porcentaje de la población activa que se puede incluir entre los voluntarios en sentido amplio. Se trata del $38 \%$, un poco más elevado que el promedio de los países en desarrollo. 
A partir de la actividad realizada, coherentemente con el modelo latino americano, las organizaciones no lucrativas peruanas se dedican, en su mayoría, a la erogación de servicios, especialmente de tipo educativo ( $75 \%$ de la ocupación), mucho más del promedio lacino americano (44\%) y mundial (30\%). Si se toma en cuenta también el trabajo de los voluntarios, destaca la importancia de iniciativas sociales como los comedores populares y el vaso de leche.

$\mathrm{La}$ preponderancia de las organizaciones que brindan servicios se repercute también sobre la composición de los recursos usados para desempeńar las actividades. Más de los dos tercios de los ingresos son contribuciones públicas y el $13 \%$ se origina de donaciones filantrópicas privadas. El modelo peruano tiene muchas similitudes con el latinoamericano, del cual difiere por una composición más equilibrada de los recursos y una incidencia más fuerte del Estado.

Desde el punto de vista de la capacidad de autosustentarse, merece subrayar las diferencias entre los sectores de actividad, mientras que educación, servicios a la comunidad y actividades culturales se financian en la casi totalidad con los ingresos originados por la venta de bienes y servicios, las demás iniciativas como las dirigidas al medio ambiente y de soporte al desarrollo, dependen de contribuciones públicas, también conseguidas por otros países en el marco de la cooperación internacional, mientras el sector salud recibe importantes donaciones privadas.

En términos generales, podemos decir que el sector no lucrativo tiene un papel importanteal interior dela sociedad y economía peruana y ha llegado a ser uno de los sectores más significativos. Sin embargo, quedan muchas debilidades. Cabe señalar la fragmentación, con la presencia de muchas organizaciones muy pequeñas que, por no alcanzar niveles adecuados de eficiencia, no dan un buen servicio a la comunidad y en lugar de promover la afirmación de las personas. 
y el desarrollo de la libertad individual, difunden en la comunidad un estilo demasiado paternalista y clientelar.

A pesar de esto, las organizaciones no lucrativas han dado y siguen brindando una gran contribución, no solo en la erogación de servicios que la población necesita mucho, sino también impulsando el cambio y contribuyendo a la creación de una sociedad más justa y democrárica, así como formando una nueva clase de líderes, muy motivados y cercanos a su comunidad.

Una investigación dela Universidad Católica Sedes Sapientiae, llevada a cabo en años recientes ${ }^{1}$ confirma la importancia del sector no lucrativo en la zona norte de la capital.

Se trata de un análisis interesante porque la zona refleja en sus características socioeconómicas el proceso de formación relativamente reciente. Gran parte de la población es el resultado de migraciones de las zonas de la sierra y de la selva, donde las precarias condiciones de vida ponían en discusión la misma posibilidad de supervivencia. Campesinos, pastores, pequeńos comerciantes, artesanos, dejaron todas sus pertenencias, rompieron con su pasado; fueron a vivir a Lima, encontraron trabajos precarios y vivieron en condiciones de vida muy pesadas en los asentamientos humanos y los pueblos jóvenes.

La categoría más adecuada para describir las condiciones de vida de Lima Norte es, sin duda, la palabra contradicción: por un lado, son evidentes las bajas condiciones de vida en comparación con el resto de Lima, pero, por otro lado, es necesario reconocer que la miseria y la desesperación no destruyeron el deseo de una vida mejor y de un protagonismo económico y social. Creatividad, laboriosidad, generosidad, una cierta capacidad de trabajar con eficacia (aun con

1 Sinell, Riccardo (coord.). Non Profit, Universita e Sviluppo. Il caso di Lima Nord. Lima: Fondo Editorial UCSS, 2005. 
escasa eficiencia); son indudablemente rasgos típicos de esta población y son la base de la difusión de iniciativas sin fines de lucro.

La investigación que ha sido desarrollada por la Universidad Catolica Sedes Sapientiae permitió conocer el rico tejido de organizaciones que operan en la zona, e hizo reflexionar sobre el papel del non profit: de este sector ya deriva una importante contribución al desarrollo y en perspectiva el impacto podría ser mayor tanto desde el punto de vista de la creación de puestos de trabajo (un ejemplo es la universidad misma que es una empresa no lucrativa, que en los cinco años de su existencia ha creado 450 puestos de trabajo), como por el desarrollo del capital humano.

Sin embargo, han sido evidenciados una serie de límites. Ante todo la informalidad que parece ser la característica fundamental, una informalidad motivada no solo por los altos costos de la formalización y por el temor que una intervención pública pueda obstaculizar la capacidad de perseguir los fines sociales, sino también, en unos casos, por la decisión de quedarse en la ambigüedad, sobretodo en materia de cómo administrar los fondos respecto de las normativas. En segundo lugar, a las actividades desarrolladas les falta eficiencia y espíritu empresarial. En tercer lugar, la orientación hacia necesidades fundamentales, en muchos casos de subsistencia, limita la actividad a la suplencia de la intervención pública insuficiente o inexistente. Por último, emerge de manera impresionante la absoluta falta de conciencia del valor de la actividad realizada, que a menudo es percibida como mera sustitución del Estado.

Frente a esto está toda la riqueza de este universo de iniciativas, la pasión, la creatividad, la generosidad, el coraje. También se ha ido notando en positivo el notable nivel de estabilidad y solidez que caracteriza a este tejido (se sigue desarrollando iniciativas sin desistir frente a las innumerables dificultades cotidianas) y también la capacidad de aurogeneración de recursos que fortalece la capacidad de 
duración en el tiempo. En efecto, no debe olvidarse que la duración es un requisito fundamental de las empresas en general y de las empresas llamadas sociales. ${ }^{\prime}$ Otro elemento interesante es la fuerte presencia de voluntarios que prestan de manera desinteresada su propio trabajo. Para el futuro, el camino iniciado por unas organizaciones no lucrativas hacia un mayor nivel empresarial es otra señal que hace bien esperar.

Al haber conocido de cerca a los protagonistas de diversas iniciativas, lo que más impresiona es la dinámica que pone en marcha la acción: los que promueven empresas de este tipo inician mirando el entorno en el cual viven a partir de un ideal, así descubren que existen necesidades insatisfechas y potencialidades no expresadas, por lo tanto, deciden obrar, sin buscar recompensas inmediatas porque lo que hacen ya tiene en sí su recompensa. Como dice Hirschman, ${ }^{2}$ se trata de una acción caracterizada por fusión de búsqueda y objetivo. El inicio a menudo no es a partir de reflexiones teóricas sofisticadas y probablemente esta es al inicio la fuerza del non profit. ${ }^{3}$ Sin embargo, el riesgo es que por falta de una visión cultural, la actividad de respuesta a necesidades concretas no despliegue todas sus potencialidades, lo que falta es una cultura de la gratuidad que nace de una cultura del trabajo, de la empresa, de la economía. Solo de esta manera, en nuestra opinión, el no profit puede ser un importante factor de desarrollo mediante un proceso de innovación social. En efecto, si se observa al modelo descrito, hay que desear que desaparezca, en el sentido que se supere el estado de indigencia que provoca su nacimiento, pero es muy importante que no desaparezca la

1 Caselut, Clara. "Non profit; una totale dignità di azienda". Persone Imprese ơ Istituzioni, $\mathrm{n}^{\circ}{ }^{\circ} 1,2000$.

2 Hirschman, A. O. Lealtd, defezione, protesta, Milano: Bompiani, 1982.

3 Ciseni, Clara «I drivers dello sviluppo del non profit nel mondow. En GASPARre, A. (ed.) Organizzazioni non profit: radici, problemi e prospettive. Genova: De Ferrari, 2002. 
riqueza que tiene por detrás y, por lo tanto, el modelo se evolucione y madure en el tiempo sin perderse y sin perder su idealidad.

Lima y más allá: la empresa no profit como factor de desarrollo

¿Qué tipo de contribución al desarrollo puede derivar del no profit? La primera consideración, casi obvia, es que el subdesarrollo, la pobreza y la miseria (no solo material) son un fenómeno negativo, sobre todo porque constituyen un límite para el pleno despliegue de la libertad y de la creatividad humana. Por tanto, superar el estado de pobreza implica poner en marcha la libertad humana y la creatividad que nace en la base de la sociedad. En este sentido, podemos decir que la libertad es el primer recurso para el desarrollo. Lo documentan muy bien unos casos que se han descubierto en la investigación: es aquello que hace que desde años las mujeres se levanten en la madrugada para preparar el desayunó a los niños de la calle o que hace decir al religioso canadiense, que ha puesto en marcha un taller / empresa para los jóvenes desempleados, donde se aprende un trabajo especializado en el sector textil y cuyos productos (tshirts) se veden a las grandes multinacionales: "Deben aprender a usar la máquina de coser como si estuviesen tocando la guitarra: esta es su obra de arte».

$\mathrm{Al}$ estudiar estos casos, se entiende que la creatividad nace de modo graruito de la libertad. Si en el caso del non profit esto es inmediatamente evidente, de hecho, no existe empresario auténtico que antes de pensar en la rentabilidad de una iniciativa no parta de la individualización de una oportunidad que la realidad ambiental le sugiere y él desarrolla (hasta llegar a la factibilidad económica), antes que nada, por un ímpetu creativo, por el deseo de dar forma o dejar huella en la realidad misma, para si mismo y para otros (los propios hijos, por ejemplo) en el tiempo. En este sentido, la lección que se 
imparte es igual para todos, no existe creatividad económica duradera ni proceso innovador auténtico que no tenga el mismo origen. ${ }^{1}$

Una segunda consideración es que la pobreza puede ser erradicada, solo si en un ámbito local se pone en marcha un mecanismo de creación de valor, no financiero, pero económico, social y humano, Crear valor, no se tiene que olvidarlo, quiere decir responder a las necesidades en modo eficaz. ${ }^{2}$ Es decir, a quien es portador de una necesidad hay que darle la capacidad de solucionarla directamente, pero esto es imposible sin la ayuda y la colaboración de los diversos actores: el desarrollo implica y requiere la solidaridad. La producción de valor en la sociedad necesica la construcción de una hilera entre los actores, que es algo más que una red porque su éxito le asegura la estricta interdependencia y complementariedad de los actores mismos en el proceso de creación de valor; esto desciende de la naturaleza misma de la actividad económica que está dramáticamente sujeta a las leyes de la escasez y del límite, por tanto, si alguno no usa bien sus recursos no solo no crea valor para sí, sino que lo destruye y lo sustrae a otros. Pues, de los contextos locales hacia aquellos globales, crear valor es una responsabilidad común.

La contribución del non profit al desarrollo se sintetiza como la capacidad de ser creador de valor de frontera: ${ }^{3}$ entre necesidades $y$ respuestas, entre profit y non profit, entre público y privado, entre las diversas formas de empresas no lucrativas, en las relaciones que cada empresa no profit establece a su interior y con el ambiente.

1 Michesin, F. Empresa y responsabilidad. Madrid: Encuentro, 1999.

2 Véase de Casszır, Clara: "Riflessioni sulla complessa tessitura del valore sociale: l'impresa sociale come "produrtore di frontiera",. EN CAMPI, S. (ed.), Impresa sociale: verso un futuro possibile. (Genova: De Ferrari, 2003), y CAsel.1, Clara "Il paradigma del valore e la sua rottura: il caso del non profio. En Impresa ProgettoRivista on line del DITEA. <www.impresaprogetto.it>, n. ${ }^{\circ} 1,2005$.

3 Véase CAsel.u, Clara "ll paradigma del valore e la sua rottura: il caso del non profito. En Impresa Progetto-Rivista on line del DITEA, 2005. 
Aplicando estas consideraciones al tema de los países en vías de desarrollo, podemos bosquejar la contribución especifica del no profit puede a la creación del valor.

\section{La frontera entre necesidades y respuestas}

A menudo, quien es portador de una necesidad no logra identificarla y comunicarla: frente al conjunto de las necesidades (viejas-nuevas; concientes-inconscientes-potenciales; satisfechasinsatisfechas-parcialmente insatisfechas) el no profit tiene una función muy importante por desarrollar para que las necesidades mismas se aclaren y se expresen. En efecto, gracias a su colocación en el medio de la sociedad, el no profit está en condiciones de crear $v o z:{ }^{1}$ si quien tiene una necesidad aprende a comunicarla y a moverse, indudablemente este es un poderoso factor de desarrollo. Además, las organizaciones sin fines de lucro son constructoras de respuestas, por ejemplo, en términos de creación de puestos de trabajo y como agentes de formación de capital humano. Es evidente el gran potencial social y económico de estas actividades, recursos que no son concientes de serlo entran en valorización, recursos que han perdido su valor lo recobran, exigencias insatisfechas encuentran el espacio para construir una respuesta, energías positivas tienen los canales para su expresión, etc. A cada una de estas observaciones corresponden casos emblemáticos de organizaciones no profit de éxito.

\section{La frontera profit / non profit}

A menudo, se trata de dos mundos con escasas relaciones entre ellos, en cambio, se pueden establecer alianzas significativas en los

1 Hirschman, A. O., op. cit. 
sectores de la producción, de la formación, de la creación de puestos de trabajo, siguiendo la misma lógica de formación de alianzas y redes que caracteriza hoy al sector privado y que evidentemente podría ser extendida a las relaciones entre los dos e incluir también el sector público, permitiendo una provechosa osmosis que puede hacer entender más fácilmente a las empresas no profit la exigencia de recorrer un serio camino empresarial (hasta adoptar el modelo de empresa social) y, al mismo tiempo, hacer crecer en las empresas profit la conciencia de la responsabilidad social. Muy interesantes en los países en vías de desarrollo son las relaciones que pueden nacer a partir del creciente interés de las grandes y medianas empresas hacia la temática de la responsabilidad social (corporate social responsibility - CSR). 'También es un modo de influencia recíproca que existan ciclos de vida de empresas que hacen el recorrido de empresas no profit a profic, así como la existencia de recorridos profesionales, sobre todo a nivel de los más altos directivos, que se desarrollan entre ambos mundos (secuencialmente o en paralelo).

\section{La frontera público/privado}

Muchas veces el no profit en los países en vías de desarrollo es débil y dependiente del sector público (a veces nacen relaciones clientelares), en cambio, hace falta romper la lógica de la subordinación para instaurar un sistema de partenariado.

Esto implica de parte del sector público el reconocimiento que el no profit no es una alternativa secundaria (second best), sino que es un modo de reinterpretar creativa y eficientemente el rol del estado de

1 Molteni, M. Responsabilità sociale e performance d'impresa. Rer una sintesi sociocompetitiva. Milano: Vita e Pensiero, 2004. 
bienestar en el contexto del welfare worth (valor social). ${ }^{\prime}$ Además, la colocación de muchas iniciativas no lucrativas muy cercanas al punto en que las necesidades nacen, lo hacen un interlocutor fundamental para el diseńo de políticas sociales (y económicas, por algunos aspectos). Un ejemplo (como ya se comentó) son las necesidades que no encuentran la manera de expresarse; otros ejemplos se refieren a la articulación de aquellas que han surgido y también a la formulación de políticas de la ocupación, para el fomento de las pequeñas y microempresas. En este sentido, el no profit puede ser un lector de necesidades, un factor de innovación en la respuesta, un vector de la subsidiariedad y un mediador entre subsidiariedad vertical y horizontal.

\section{La frontera al interior del grupo de las empresas no lucrativas}

Justamente por la inadecuada conciencia de su valor que a menudo caracteriza a las iniciativas no profit en los paises en desarrollo, no siempre existen relaciones entre las diferentes iniciativas y a veces hay competencia entre ellas (sobretodo cuando se compite para conseguir los escasos recursos disponibles); viceversa, una fuerte capacidad de aliarse, ayudarse recíprocamente y hasta conformar grupos de presión (lobbying), podría ser extremadamente importante para toda la sociedad. Hace falta poner en acción relaciones que produzcan valor: entre estas son importantes aquellas que tienen el fin de fomentar $y$ fortalecer el espíritu emprendedor $y$, a nivel general, aquellas que buscan reformular su propia misión a la luz de los cambios ambientales y de las exigencias de producir valor en modo eficiente. Un tema crucial es también aquello de la creación de nuevos mercados, construidos según una lógica no individualista. Entre los ejemplos significativos en esta dirección, recordaremos, por su relevancia en los países en

1 Giorgetti, G. «Per uno stauto dell'impresa socialen, En Campl, S. (ed.). Imprea sociale: verso un futuro possibile. Genoya: De Ferrari, 2003. 
desarrollo, la tentativa de diseñar relaciones internacionales con un fuerte contenido ético así como la construcción de mercados de vouchers.

\section{La frontera interna a cada organización non profit}

Las fronteras no están solo al externo, también están al interno de las estructuras del non profit. No solo porque el confín interno/externo se atenúa $y$, por lo tanto, una producción de valor que se realizaba toda al interno de la empresa se mueve hacia otros sujetos y viceversa, sino sobre todo por medio de la cultura empresarial, en efecto, la cultura tiene muchos reflejos sobre la capacidad de satisfacer las exigencias de todos los que operan en la organización $y$, además, es propuesta hacia el exterior de un modo de concebir la actividad económica. Nos estamos refiriendo al tema de las relaciones con los trabajadores, el voluntariado, además de la estructura organizativa. Desde este punto de vista el no profit puede ser una escuela o una fábrica de capital humano, donde pueden crecer empresarios y agentes económicos en grado de conjugar idealidad con profesionalidad y eficiencia. Están también colocadas en este punto todas las temáticas de espíritu emprendedor. La contribución decisiva que este mundo puede dar al desarrollo pasa por medio de un proceso de maduración de las formas existentes y de evolución mediante formas empresariales orientadas hacia un sentido social. Desde esta perspectiva, la empresa social constituye un deseable punto de llegada del proceso de evolución.

En conclusión, el reto que las organizaciones no lucrativas tienen es la capacidad de contribuir al proceso de desarrollo. La conveniencia de este sector de la sociedad se tiene que evaluar justamente a partir de esto. En detalle, se pueden identificar los siguientes puntos:

1 CASELLI, Clara. «Etica e responsabilità d'impresa nelle relazioni internazionali», Symphonya, n. ${ }^{\circ}$ 1, 2003. 
- la capacidad de crear "voz";

- la capacidad de crear iverdaderos" puestos de trabajo;

- la capacidad de contribuir al desarrollo del capital humano;

- la capacidad de ser constructores de alianzas para crear valor;

- la capacidad de transformarse hacia formas de empresas sociales.

Como es evidente, se trata de un desafío no de hace poco, pero no imposible, como diversas experiencias en muchos países del mundo demuestran.

La cosa más importante, que no se tiene que olvidar nunca, es cuán inestimable es la existencia de iniciativas capaces de construcción a partir de la gratuidad, porque recuerda a todos que sin un valor sobre el cual construir no existen valores, pero tampoco existe valor económico y entonces de la pobreza —en todas sus variadas formasno se puede salir. 


\section{EMPRESAS NO LUCRATTVASY DESARROLLO}

\section{Bibliografia}

Caselli, C. "Il non profit; aspetti aziendalim. Persone of Imprese, n. ${ }^{\circ} 2,1998$.

—Non profit: una totale dignità di azienda». Persone Imprese \& Istituzioni, $\mathrm{n} .^{\circ} 1,2000$.

—I drivers dello sviluppo del non profit nel mondo". En Gasparre, A. (ed.). Organizzazioni non profit: radici, problemi e prospettive, Genova: De Ferrari, 2002.

"Etica e responsabilità d'impresa nelle relazioni internazionali». Symphonya, n. ${ }^{\circ}, 2003$.

"Riflessioni sulla complessa tessitura del valore sociale: l'impresa sociale come "produttore di frontiera" $"$. En CAMPI, S. (ed.). Impresa sociale: verso un futuro possibile. Genova: De Ferrari, 2003.

- "Il paradigma del valore e la sua rottura: il caso del non profit". En Impresa Progetto-Rivista on line del DITEA. <www.impresaprogetto. it>, n. ${ }^{0} 1,2005$.

CODA, V. «Lorientamento strategico dell'impresa», En BLANCHI T.; V. Coda; G. Mazza; O. Paganelli; G. Pellicelli (eds.). Trattato di Economia Aziendale. Torino: UTET, 1988.

Giorgetti, G. «Per uno statuto dell'impresa sociale». En Campi, S. (ed.). Impresa sociale: verso un futuro possibile. Genova: De Ferrari, 2003.

Hirschman, A. O. Lealtà, defezione, protesta. Milano: Bompiani, 1982.

Micheun, F. Empresa y responsabilidad. Madrid: Encuentro, 1999. 


\section{CLARA CASELLI}

Molteni, M. Responsabilità sociale e performance d'impresa. Per una sintesi socio-competitiva. Milano: Vita e Pensiero, 2004.

Portocarrero, F:; C. Sanborn. "Entre el estado y el mercado: definiendo el sector sin fines de lucro en el Perún. Apuntes 43, n. 2, 1999.

Portocarrero, F; C. Sanborn; H. Cueva; A. Millán. Más allà del individualismo: el tercer sector en el Perú. Lima: Centro de Investigación de la Universidad del Pacífico, 2002.

SALAMON, L. M.; H. K. ANHEIER. Il settore non profit in un confronto internazionale: profili e tipi. Milano: Università Cattolica del Sacro Cuore, Centro di ricerche sulla cooperazione, Quaderni occasionali, n. 3, 1994.

Il settore non profit in una prospettiva comparata. Una panoramica. Milano: Università Cattolica del Sacro Cuore, Istituto per la ricerca sociale, Quaderni occasionali, n. ${ }^{\circ}$ 6, 1994.

Global Civil Society, Dimensions of the Non Profit Sector. Baltimore: The Johns Hopkins Center for Civil Society Studies, MD, 1999.

Salamon, L. M.; S. W. Sokolowski; R. List. Global Civil Society. An Overview. Baltimore: The Johns Hopkins Center for Civil Society Studies, MD, 2003. 